\title{
LA RECONFIGURACIÓN DEL MEDIO RURAL EN GALICIA (ESPAÑA): algunas reflexiones acerca de su enseñanza en la sociedad postindustrial
}

\author{
FRANCISCO XOSÉ ARMAS QUINTÁ
}

Doutor em Geografia. Professor Interino da Universidade de Santiago de Compostela. Grupo de Pesquisa Analise Territorial (ANTE). Researcher ID: H-4343-2015. ORCID: https://orcid. org/0000-0002-8644-8185 E-mail: franciscojose.armas@gmail.com

\section{FRANCISCO RODRÍGUEZ LESTEGÁS}

Doutor em Geografia e História (Seção de Geografia). Catedrático de Universidade de Didática das Ciências Sociais. Universidade de Santiago de Compostela. Grupo de Pesquisa Razoamento,

Discurso e Argumentação (RODA). Researcher ID: A-2360-2015. ORCID https://orcid. org/0000-0002-1733-3085_E-mail: f.lestegas@usc.es 


\section{XOSÉ CARLOS MACÍA ARCE}

Doutor em Geografia. Professor Interino da Universidade de Santiago de Compostela. Grupo de Pesquisa Razoamento, Discurso e Argumentação (RODA). ORCID: https://orcid.org/00000001-8597-4557 E-mail: xosecarlos.macia@gmail.com

\section{RESUMEN}

La inmersión en la sociedad de la información, entre otros factores, está teniendo un importante impacto a nivel social, económico, cultural, etc. y las áreas rurales no han quedado al margen de este proceso. Desde la segunda mitad del pasado siglo el medio rural en Galicia ha iniciado un fuerte proceso de desagrarización acompañado de intensos procesos migratorios, bien hacia el extranjero (Latinoamérica y Europa), bien hacia otros destinos nacionales y regionales. La progresiva terciarización de la economía del medio rural en esta región, unido a la difusión de las tecnologías de la información y de la comunicación, está configurando diferentes ruralidades que el alumnado debe conocer. En el presente artículo se estudia cómo se está llevando a cabo la enseñanza del medio rural en Galicia en las aulas de bachillerato revisando, de un lado, los contenidos curriculares, y del otro, diversos libros de texto publicados por algunas de las editoriales más relevantes del país.

Palabras clave: Medio rural. Tecnologías de la Información y de la Comunicación. Bachillerato. Currículo. Libro de Texto.

\section{A RECONFIGURAÇÃO DO MEIO RURAL NA GALÍCIA (ESPANHA): algumas reflexões sobre o seu ensino na sociedade pós-industrial.}

A imersão na sociedade da informação, entre outros fatores, está tendo um impacto importante aos níveis social, econômico, cultural, etc., e as áreas rurais não ficaram à margem desse processo. Desde a segunda metade do século passado, o meio rural na Galícia, iniciou um forte processo de desagregação acompanhado de intensos processos migratórios, tanto para o exterior (América Latina e Europa), quanto para outros destinos nacionais e regionais. A terceirização progressiva da economia do meio rural nesta região, juntamente com a difusão das tecnologias de informação e comunicação, estão configurando diferentes ruralidades que os estudantes devem conhecer. Este artigo estuda o ensino do meio rural da Galícia na Educação secundária, analisando por um lado os conteúdos curriculares e, por outro, vários livros texto publicados por algumas das editoras mais relevantes do país.

Palavras chave: Meio rural. Tecnologias da Informação e Comunicação. Educação Secundária. Currículo. Livro Texto. 


\section{RECONFIGURATION OF RURAL AREAS IN GALICIA (SPAIN): some thoughts on teaching in the postindustrial society}

Immersion in the information society, among other factors, is having a noteworthy impact at social, economic and cultural level, and rural areas have not been excluded from this process. Since the second half of the last century, rural areas of Galicia have begun a considerable de-agrarization process accompanied through intense migratory processes, either by going abroad (Latin America and Europe), or to other national and regional destinations. The progressive outsourcing of the economy of rural areas, coupled with the spread of information and communication technologies, is configuring several ruralities that the students must know. In this article, on the one hand, the teaching of rural areas in Galicia in the Spanish baccalaureate classrooms is studied, and on the other hand, several textbooks published by some relevant publishers from the country are analyzed.

Keywords: Rural Areas. Information and Communication Technologies. Spanish baccalaureate. Curriculum. Textbook.

\section{LA RECONFIGURACIÓN DEL MEDIO RURAL EN GALICIA (ESPAÑA): algunas reflexiones acerca de su enseñanza en la sociedad postindustrial}

En las áreas rurales de los países desarrollados han tenido lugar, en las últimas décadas, cambios tan trascendentales como incuestionables y que dieron lugar a una gran diversidad que complica la ya difícil tarea de su definición y delimitación. Estas transformaciones, que han tenido lugar de forma progresiva, llevaron a que en estas áreas coexistan diversos grados de ruralidad en función de distintos factores diversos como la importancia del papel de la agricultura, el grado de desarrollo de la pluriactividad y diversificación económica, o el propio desarrollo urbano (Sancho y Reinoso, 2012). La descentralización de la residencia y la proliferación de otras actividades productivas distintas de las agrarias, como la industria y una amplia gama de servicios, provocaron que el medio rural se convirtiese en un complemento de las áreas urbanas, siendo las diferencias entre estas dos realidades cada vez menos visibles (García, Tullas y Valdovinos, 2010).

Los cambios que tuvieron lugar en el medio rural desde la década de los años noventa del pasado siglo fomentaron el surgimiento de una ruralidad diferente a la tradicional (Rubio, 2010). El espacio rural de la sociedad postindustrial muestra una complejidad de usos y funciones que se contrapone con la polarización claramente definida entre el campo y la ciudad de la primera 
mitad del siglo XX (García, Tullas y Valdovinos, 2010). Esta evolución hace que el medio rural comience a ser visto no como una simple superficie de producción, sino como un espacio que aporta una serie de valores de elevado interés para una sociedad globalizada y cada vez más urbana (Armesto, 2005).

El concepto de lo rural ha pasado, en las últimas décadas, de ser sinónimo de tosco y atrasado a tener connotaciones positivas asociadas a la calidad (Sancho y Reinoso, 2012). Estos cambios supusieron una alteración considerable de este concepto hasta el punto de que algunos autores asintieran que la extensión del modo de vida urbano al rural estuviese borrando algunas de sus particularidades más significativas como es el modo de vida campesino (Molinero y Alario 1994). Pese a esto, en la literatura científica se pueden encontrar posturas donde se defiende que la agricultura sea la actividad esencial para preservar un territorio rural activo. Si la agricultura desaparece del medio rural este dejaría de serlo, corriendo el riesgo de que se convierta en un hábitat urbano o periurbano con población dispersa, o bien en un territorio humanamente desertizado (MAPA, 2003; Hervieu, 1995).

Existe una tendencia a generalizar acerca de los problemas "estándar" del medio rural, tales como el declive económico y demográfico, la agricultura marginal, o la situación periférica de una buena parte de estos espacios, asumiendo que estos son homogéneos (McDonag, 1998). Pero la realidad es que el sistema productivo del medio rural se ha ido reestructurando hasta el punto en el que lo rural ya no es sinónimo de agrario, y los asentamientos avanzaron, en unos casos, hacia el declive económico y demográfico y, en otros, hacia la urbanización, como ocurre en las proximidades de las áreas metropolitanas (Rubio, 2010). Estos aspectos llegan, en muchos casos, a diluir las fronteras entre lo urbano y lo rural dificultando su delimitación (Sancho y Reinoso, 2012).

Se ha escrito mucho sobre el concepto de ruralidad y también desde puntos de vista muy diferentes. Se trata de un debate muy antiguo en donde se cuestiona si el concepto "rural" es un concepto geográfico, una localización con límites establecidos sobre un mapa, una representación social, una comunidad con intereses similares, o una cultura y forma de vida (Plessis et al., 2001). Este concepto es utilizado tanto para comparar diferentes paisajes, como para diferenciar distintos escenarios sociales y económicos (Pratt, 1996).

Una buena parte de las definiciones que se han propuesto para definir el medio rural están fundamentadas en base a su dificultad por delimitarlas debido a la creciente rururbanización, haciendo que las fronteras entre campo y ciudad sean cada vez más difusas (Entena-Durán, 2012). Las áreas rurales cumplen nuevas funciones en la sociedad postindustrial, ofreciendo nuevas 
localizaciones para las actividades de los sectores industrial y de servicios. Del mismo modo, la desconcentración de la población urbana y la descentralización de las actividades económicas inyectan, en mayor o menor medida, dinamismo a los espacios rurales que están más próximos (en tiempo o en espacio) a las ciudades. Por otro lado, las áreas rurales nutren a las ciudades de población joven que forman parte de un éxodo rural (García, Tullas y Valdovinos, 2010) que difiere, considerablemente, del que tuvo lugar en la segunda mitad del pasado siglo.

Después de casi un siglo de importantes esfuerzos para definir el medio rural no existe, aún, una definición objetiva y ampliamente aceptada del término (Paniagua y Hoggart, 2002; Calatrava y Melero, 2003) debido a la diversidad de los elementos que lo forman (Lázaro, 1995; Márquez, 2002). Tradicionalmente el medio rural se definía como el espacio físico y social de la actividad agraria, identificando lo rural con lo agrario (Florencio, 2000). Pero existe una gran variedad de posturas en la literatura científica al respecto. Algunos autores como Lázaro (1995), Ceña (1995) y Guibertau (2002) sostienen que el medio rural es un territorio que ofrece materias primas y recursos naturales y donde se desarrollan una amplia gama de actividades económicas, aunque creen necesario diferenciar el medio rural de la producción agraria puesto que, aunque guardan relación entre sí, tienen un significado diferente.

Otros, en cambio, sostienen que es necesario definir un nuevo papel para estos espacios que pasa por el cuidado y la preservación del entorno natural (Ramos y Romero, 1995). El creciente interés social por el enfoque medioambiental se ha intensificado en las investigaciones de la geografía rural destacando los valores paisajísticos y simbólicos, además de poner atención en la degradación del medio ambiente y en el carácter depredador de la agricultura moderna sobre los recursos no renovables. La población agraria debe caminar hacia una combinación de su función productora de alimentos con la de cuidar y mantener el patrimonio natural, cultural y arquitectónico, además de orientar el modelo de la agricultura intensiva hacia una agricultura más extensiva, incorporando nuevas técnicas que contribuyan a preservar el medio ambiente (García, Tullas y Valdovinos, 2010).

La dificultad de definir y delimitar el medio rural se agudiza cada vez más, si cabe, con la difusión de la sociedad de la información. Las nuevas tecnologías han regenerado la interconexión entre lo rural y lo urbano, facilitando la instalación de nuevas actividades y servicios (Moyano, 2000). A ello también han contribuido la creación de infraestructuras de comunicaciones (vías de alta capacidad, tren de alta velocidad) disminuyendo las distancias, permitiendo así una mayor integración del territorio y pasando del contraste a la gradación espacial (Robinson, 1990). 
El proceso de desconcentración demográfica y económica de la ciudad hacia el campo, promovido por las tecnologías de la información y de las comunicaciones, hacen posible volver al modelo donde el lugar de trabajo coincidía con el lugar de residencia (García, Tullas y Valdovinos, 2010). El hogar pasa a ser un lugar donde relax, ocio y descanso dejan de ser exclusivos y adquieren nuevas funciones como lugar de trabajo, formación, etc. (Cairncross, 2001).

La terciarización del mundo rural es la clave que define a estos espacios en la actual sociedad postindustrial (Rubio, 2010), así como la transición hacia el paradigma postproductivista donde deben enmarcarse las estrategias de desarrollo rural. Todos estos aspectos deben ser estudiados en las aulas de bachillerato en España para que los alumnos adquieran un conocimiento global de la sociedad y, en especial, del medio rural y su nueva función de cara a preservar y cuidar el medio natural.

\section{La integración del medio rural en el currículo de bachillerato en España}

Los contenidos que se imparten en bachillerato en España, y más en concreto en la Comunidad Autónoma de Galicia, están regulados por el Real Decreto 1105/2014, de 26 de diciembre, donde se recoge el currículo básico de la Educación Secundaria y Obligatoria y del Bachillerato, que elabora el Ministerio de Educación, Cultura y Deporte del Gobierno de España. A su vez, la Consellería de Cultura, Educación y Ordenación Universitaria del gobierno regional de la Xunta de Galicia recoge los contenidos curriculares impartidos en su comunidad autónoma. En este caso, los contenidos curriculares para el bachillerato están regulados por el Decreto $86 / 2015$, de 25 de junio, por el que se establece el currículo de la Educación Secundaria Obligatoria y del Bachillerato en la Comunidad Autónoma de Galicia. Estos decretos están vinculados con la Ley Orgánica 8/2013, de 9 de diciembre, para la Mejora de la Calidad Educativa (LOMCE).

El bachillerato, tal y como recogen los dos decretos apuntados anteriormente, tiene como finalidad "proporcionar al alumnado formación, madurez intelectual y humana, conocimientos y habilidades que le permitan desarrollar funciones sociales e incorporarse a la vida activa con responsabilidad y competencia" (Real Decreto 1105/2014, p. 187; Decreto 86/2015, p. 25.46525.466). Abarca dos cursos y existen tres modalidades: ciencias, humanidades y ciencias sociales, y artes. En el caso de la modalidad de humanidades y ciencias sociales, los alumnos pueden optar por el itinerario de humanidades o por el itinerario de ciencias sociales.

La materia de Geografía se imparte, como materia optativa troncal, en el itinerario de ciencias sociales. Tal y como recoge el currículo, tiene como objetivo "la comprensión del territorio [...] y que el alumnado pueda explicar la realidad geográfica española, con especial 
atención al territorio gallego" (Decreto 86/2015, p. 26.449). Con la finalidad de alcanzar este objetivo, los contenidos, los criterios de evaluación y los estándares de aprendizaje se organizan en doce bloques temáticos: la geografía y el estudio de espacio geográfico; el relieve español y su diversidad geomorfológica; la diversidad climática y la vegetación; la hidrografía; los paisajes naturales y las relaciones entre naturaleza y sociedad; la población española; el espacio rural y las actividades del sector primario; las fuentes de energía y el espacio industrial; el sector servicios; el espacio urbano; formas de organización territorial; y, por último, España en Europa y en el mundo.

El currículo dedica, en la materia de Geografía impartida en el segundo curso del bachillerato, un bloque temático que lleva por título "El espacio rural y las actividades del sector primario". El propio título avanza la importancia que se le atribuye al sector primario a la hora de estudiar el medio rural y que se constata una vez revisados los contenidos que se recogen en este bloque temático. De los ocho temas en los que se distribuyen los contenidos, todos están orientados al sector primario. Entre ellos se pueden citar los que tratan el estudio de las actividades agropecuarias, forestales y pesqueras y su peso en el PIB; los paisajes agrarios, su morfología y estructura; la estructura de la propiedad y tenencia de la tierra; las explotaciones agrarias, tipos y características; la política agraria común, así como las actividades pesqueras y sus características. Todos los contenidos que se proponen en el currículo giran en torno al sector primario, con especial atención a la agricultura y ganadería.

Resulta curioso y, a la vez preocupante, que el término "rural" solo aparezca en tres ocasiones en todos los contenidos curriculares de la materia de Geografía que se imparte en el segundo curso de bachillerato. La primera vez que aparece el término rural es en el propio título del bloque temático: "El espacio rural y las actividades del sector primario". En las otras dos ocasiones aparece en los criterios de evaluación de los temas tres y ocho, donde se estipula como criterios de evaluación, "analizar adecuadamente un paisaje rural..." (Decreto 86/2015, p. 26.463) y "obtener y seleccionar información de contenido geográfico relativo al espacio rural..." (Decreto $86 / 2015$, p. 26.464). No hay ninguna referencia a este término en los contenidos de los ocho temas que componen el bloque dedicado al estudio del medio rural en la materia de Geografía lo que, a nuestro juicio, es una gran carencia que debería ser solventada.

La revisión curricular para la materia de Geografía revela, desde nuestro punto de vista, una visión arcaica y anacrónica del medio rural, tanto en Galicia como en España. La selección de contenidos dedicados a estudiar casi en exclusiva el sector primario, ofrece una perspectiva de la realidad del medio rural poco realista, desvirtuada y cada día más alejada de la realidad. Según los datos que publica el Instituto Galego de Estatística (la oficina estadística regional oficial), a 
comienzos de los años noventa del pasado siglo, para el conjunto de Galicia el 20\% de la población ocupada trabajaba en el sector primario y en el $60 \%$ de los municipios de esta región, al menos tres de cada diez gallegos estaban empleados en el sector primario. Por aquel entonces había muchos municipios donde la mayoría de la población ocupada trabajaba en el sector primario llegando, en algunos de ellos, a alcanzar valores superiores al $80 \%$. En lo que respecta al sector servicios, en estos años solo el $7 \%$ de los municipios gallegos ocupaba, al menos, a la mitad de su población en este sector. Veinte años más tarde, el mismo organismo revelaba datos muy dispares. La población ocupada a nivel regional en el sector primario bajó del $20 \%$ al $7 \%$ y el número de municipios que ocupaba a la mitad o más de su población en el sector servicios pasó, en este período, del 7\% al 70\%. Estos datos evidencian que el medio rural es ya algo más que agricultura y ganadería y, desde nuestro punto de vista, son necesarios cambios considerables, cuando no radicales, en los contenidos curriculares para poder enseñar las realidades actuales del medio rural del siglo XXI.

Uno de los aspectos que más llama la atención en los contenidos curriculares, para la materia de Geografía, es la total ausencia del concepto "rural" y las dificultades que existen para definirlo y delimitarlo. El alumnado debe conocer esta realidad y, sobre todo que, pese a los numerosos intentos de delimitarlo y definirlo a lo largo de las últimas décadas, aún hoy día no existe un consenso en la comunidad científica al respecto. Ello evidencia la complejidad de este proceso fruto de la gran diversidad de estos espacios y las múltiples variables que deben ser analizadas. Esta situación se ve acentuada con la progresiva difusión de las tecnologías de la información y de las comunicaciones y los servicios y oportunidades que estas ofrecen para las áreas rurales.

Cada día es más complejo delimitar lo rural de lo que no lo es ya que, tal y como señala Robinson (1990), la creación de infraestructuras, bien sean terrestres o bien de telecomunicaciones, disminuyen las distancias, permiten una mayor integración del territorio y, como consecuencia, se pasa del contraste a la gradación espacial. En el caso de Galicia, se puede hablar de diferentes ruralidades con características muy particulares y que el alumnado debe conocer. A lo largo de las últimas décadas en el medio rural se han ido implantando actividades que nada o poco tienen que ver con el sector primario. En el proceso de inmersión en la sociedad de la información, las áreas rurales están siendo el escenario de nuevas actividades económicas en las que están muy presentes las nuevas tecnologías. El comercio electrónico va a tener una gran expansión dado que Internet será el mayor canal de difusión y distribución mundial de bienes y servicios, y las áreas rurales no van a quedar al margen de este proceso. Autores como Drucker (2001) afirman que el comercio electrónico es, en la sociedad de la información, lo que fue el ferrocarril en la sociedad 
industrial. En este escenario, donde las distancias son eliminadas, las áreas rurales tienen acceso, al igual que las ciudades, a ese mercado global donde la competencia ya no tiene fronteras.

La revolución de las tecnologías de la información y de las comunicaciones hacen posible la instalación de nuevas actividades económicas en el medio rural (empresas de diseño gráfico, software, etc.), ya que la desaparición de las distancias hace posible la deslocalización de muchas de las actividades que antes se llevaban a cabo en las ciudades. El atractivo de las áreas rurales pasa por un menor coste del precio del suelo, mano de obra cualificada y más barata que en entornos urbanos, incentivos fiscales, mayor calidad de vida, etc.

Estos aspectos deberían estar presentes en los contenidos curriculares en la materia de Geografía. Si el objetivo principal que persigue el currículo para esta materia es la comprensión del territorio y que el alumnado pueda explicar la realidad geográfica gallega, difícilmente podrá ser alcanzado si no se tiene en cuenta la amplia gama de actividades económicas que están teniendo lugar en este territorio y, en especial, en el medio rural. Son muchas las transformaciones que están teniendo lugar en estas áreas a raíz de la difusión de la sociedad de la información. Estos cambios son tan importantes como complejos por lo que es fundamental que sean analizados, enseñados y aprehendidos en las aulas.

\section{El medio rural en los libros de texto.}

Para completar el análisis acerca de cómo se está llevando a cabo la enseñanza del medio rural en las aulas de bachillerato en Galicia, además de revisar los contenidos que recoge el currículo, se examinaron las propuestas de tres editoriales diferentes que, a su vez, abarcan las tres últimas leyes educativas: la Ley Orgánica 1/1990, de 3 de octubre, de Ordenación General del Sistema Educativo (LOGSE); la Ley Orgánica 2/2006, de 3 de mayo, de Educación (LOE); y la Ley Orgánica 8/2013, de 9 de diciembre, para la mejora de la calidad educativa (LOMCE). Los manuales que se seleccionaron, para la materia de Geografía de segundo curso de bachillerato, fueron: Grupo Anaya (2001) estando vigente la LOGSE; Vicens Vives (2009) estando vigente la LOE; y, por último, el manual publicado por Anaya (2016) estando vigente la actual ley, la LOMCE. La finalidad de esta selección no fue otra más que examinar cómo se han ido abordando los contenidos curriculares referidos al estudio del medio rural desde el cambio de siglo hasta nuestros días.

La editorial Anaya (2001) organizaba los contenidos para la materia de Geografía en cuatro bloques temáticos: el medio físico español, la ocupación y el poblamiento, las actividades 
económicas y la organización del territorio, y la proyección internacional de España. Los cuatro bloques temáticos contienen las once unidades didácticas en las que se agrupan los contenidos de la materia, estando una de ellas dedicada a estudiar el "Poblamiento rural y urbano" y otra específica en donde se abordan "Las actividades del sector primario". En la primera de ellas, los contenidos están diseñados para estudiar tanto el espacio rural como el urbano, si bien al primero se le dedican siete páginas y al segundo cuarenta y tres. Desde nuestro punto de vista, y con independencia de la descompensación temática en esta unidad didáctica, tanto el estudio del medio rural como el del espacio urbano requerirían, cuando menos, una unidad didáctica propia.

Los contenidos que se recogen para el medio rural en esta unidad, compartida con el espacio urbano, son muy limitados y quedan restringidos al tipo de poblamiento y hábitat. El primer aspecto que se plantea y que se resuelve en un único párrafo es, precisamente, la delimitación del medio rural, uno de los temas más complejos y sobre el cual aún no hay un consenso en la comunidad científica. Aunque siendo conscientes de que es necesario establecer algún criterio para la delimitación urbano rural, consideramos que, cuando menos, debería plantearse esta cuestión y exponer algunos ejemplos de delimitaciones y variables utilizadas, así como las ventajas e inconvenientes de cada uno de ellas.

En el caso de este manual, el planteamiento que se expone en lo que se refiere a la delimitación urbano rural resulta confuso. De un lado, se indica que "El poblamiento rural está integrado por los núcleos de población menores de 10.000 habitantes" (Muñoz, 2001, p. 187) mientras que en el siguiente epígrafe se señala que "El censo considera núcleos rurales en sentido estricto a los inferiores de 2.000 habitantes" (Muñoz, 2001, p. 187). Después de leer estos dos enunciados es probable que al alumnado no le quede muy claro cuál es el criterio que delimita estos espacios y, en definitiva, qué espacios son considerados rurales y urbanos. Esa duda es extensible a cualquier investigador o lector dado que no queda claro ni la unidad administrativa a la que se refiere (municipio, núcleos, entidades singulares, entidades colectivas) ni el umbral demográfico a partir del cual se considera rural o urbano.

Además de esta cuestión, en las siete páginas que dedican a este tema solo se hace alusión a las diferentes tipologías del poblamiento rural, así como a los cambios recientes que han tenido lugar en estos espacios. A este aspecto, que a nuestro juicio es el tema fundamental que explica la configuración actual del medio rural en Europa, se le dedica media página. En estas líneas simplemente se hace alusión al éxodo rural iniciado en la década de los años sesenta del pasado siglo, y a algunas actividades que se impulsaron como la industrialización endógena o el turismo rural. Sin duda, es un planteamiento muy limitado y que no explica ni los cambios que tuvieron lugar en las últimas décadas, ni la configuración actual del medio rural europeo. 
El diseño de una unidad específica dedicada a las actividades del sector primario indica la fuerte asociación que existe, aún, entre lo rural y lo agrario. Y este aspecto queda muy patente en los contenidos que se proponen en este manual dado que se le dedican más de cincuenta páginas. Se podría afirmar que esta editorial centraliza todo lo relacionado con el mundo rural con las actividades del sector primario.

En esta unidad se recogen aspectos tales como los factores físicos y humanos de la actividad agraria, la estructura agraria, la política agraria antes y después de que España entrase a formar parte de la Comunidad Europea, los usos del suelo agrario, la producción ganadera, la producción forestal, los problemas de la actividad agraria, la ordenación del espacio rural, los paisajes agrarios de España, la actividad pesquera y su estructura, la producción pesquera, los problemas de la actividad pesquera, la política pesquera, y la acuicultura. Todos estos aspectos tienen interés, pero a nuestro modo de ver, deberían ser tratados con un enfoque más sintético y relacionados con las nuevas actividades que están teniendo lugar en estos espacios fruto, en gran medida, de la difusión de las nuevas tecnologías.

En lo referente a las actividades propuestas, en la primera unidad didáctica están todas ellas orientadas a la parte dedicada al estudio del poblamiento urbano. En el segundo caso, la unidad didáctica que estudia las actividades del sector primario, predominan las actividades que se asocian con el modelo didáctico tradicional o transmisivo, donde se enseña una geografía descriptiva y memorística en lugar de promover una geografía activa, reflexiva y crítica (Macía, Rodríguez y Armas, 2015). Son algunos ejemplos donde se le pide al alumnado que defina parcela, barbecho, agricultura intensiva, etc. (Muñoz, 2001, p. 298), o bien completar cuadros resumen o cuadros comparativos - Figura 1.

Figura 1: Cuadro resumen: la política agraria y la política pesquera de la Unión Europea

\begin{tabular}{|c|c|c|c|c|}
\hline \multirow[t]{2}{*}{ UE } & \multicolumn{3}{|c|}{ Politica agroria } & \multirow[t]{2}{*}{ Politica pesqueiro } \\
\hline & Aaricole & Gandeire & Forestal & \\
\hline Obxectives & & & & \\
\hline Achuocibest & & & & \\
\hline $\begin{array}{l}\text { Repercuvide: } \\
\text { en Esponte }\end{array}$ & & & & \\
\hline
\end{tabular}

Fuente: Muñoz, 2001. 
La editorial Vicens Vives (2009) estructura los contenidos de la materia en cuatro bloques temáticos: naturaleza y medio ambiente, territorio y actividades económicas, población y sistema urbano, y España en una Europa y mundo globales. En estos cuatro bloques se distribuyen las dieciséis unidades didácticas en las que organizan los contenidos y, de las cuales ninguna de ellas está dedicada específicamente a estudiar el mundo rural. Solamente proponen un único tema de los dieciséis para el estudio de los "Cambios y permanencias en el sector primario". No ocurre lo mismo con respecto a los espacios urbanos ya que, en este caso, se le dedican dos temas específicos.

Los contenidos que abordan en esta unidad didáctica están todos asociados, como bien refleja su título, a estudiar el sector primario en España. Están organizados en torno a cuatro ejes temáticos: de la agricultura tradicional a la agricultura de mercado, producción y comercialización agrarias, los paisajes rurales de España, y la pesca en España. Esta selección de contenidos sigue la misma línea que marca el currículo al centralizar el estudio del medio rural con el sector primario.

En el primer eje temático se recogen diversos aspectos que explican la transición de la economía de subsistencia a la economía de mercado, como la capitalización del campo, la evolución de la superficie agraria, y la estructura de las explotaciones agrarias. En el segundo eje temático hacen alusión, igual que en la editorial Anaya, (2001) a la Política Agraria Común, si bien de un modo mucho más sintetizado, a la producción ganadera y la comercialización de los productos agrarios. En el caso de los paisajes agrarios de España, se presentan cinco tipologías: el paisaje agrario atlántico, el paisaje agrario mediterráneo, el paisaje agrario de interior, el paisaje agrario de montaña, y el paisaje agrario de Canarias. Por último, este tema cierra los contenidos con un epígrafe dedicado a la actividad pesquera haciendo alusión a los caladeros de los barcos de pesca españoles y la política pesquera comunitaria.

Al no contemplar ninguna unidad didáctica específica dedicada al medio rural, no se hace alusión a su definición y delimitación, y en especial, a los profundos cambios que han tenido lugar en las últimas décadas en estos espacios, más allá de los que acontecieron en el sector primario. En el medio rural actual la agricultura ya no es una actividad exclusiva, sino que conviven muchas otras que, otrora, eran exclusivas del ámbito urbano. Las nuevas tecnologías hicieron posible la desaparición de las distancias y, con ellas, muchas de las dificultades que condicionaron el desarrollo y el futuro del medio rural. Todos estos aspectos deben ser enseñados y trabajados en las aulas de bachillerato con el objetivo de que los discentes perciban las nuevas realidades y también las nuevas oportunidades del medio rural del siglo XXI. 
En cuanto a las actividades propuestas, teniendo en cuenta las limitaciones de los contenidos, se percibe que se apuesta por una enseñanza de la geografía activa, reflexiva y crítica, con lo que el resultado de estas provocará la reflexión, la actitud crítica y la interpretación y relación de conceptos (Macía, Rodríguez y Armas, 2016; Souto, 1998). Un ejemplo de ello se observa en una de las actividades donde los discentes deben analizar los cambios que tuvieron lugar en una finca en un período de veinticinco años comparando dos planos. En este ejercicio se proporciona a los alumnos una información específica sobre la finca en cada fecha y, en base a esta, deben comentar los cambios de titularidad, los cambios en la explotación de la tierra y en el trabajo, en la parcelación, los cultivos, etc. - Figura 2.

Figura 2: Paso de una economía tradicional a una economía de mercado

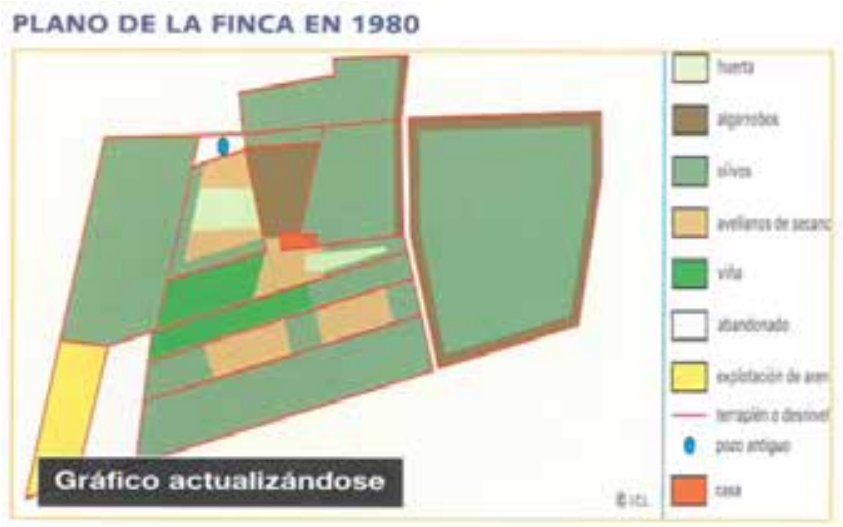

PLANO DE LA FINCA EN 2005

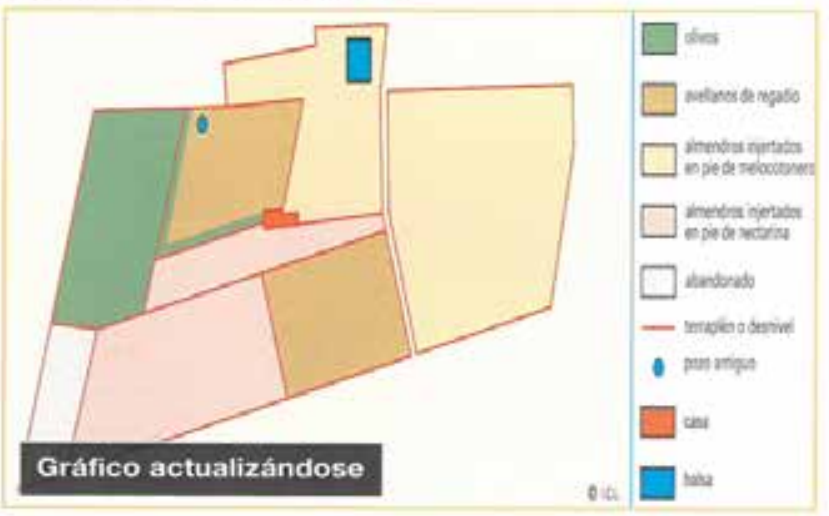

Información sobre la finca en 1980

Explotación agraria situada en la costa mediterrảnea con una extensión de 16 ha. El propietario es un médico que la heredó y vive de su profesión en la ciudad. Ni a él ni a sus hijos les interesa la tierra y la agricultura, pero mantiene la propiedad porque es un patrimonio que va aumentando de valor

Un aparcero trabaja la tierra y reside en la finca. El aparcero reparte la cosecha o los beneficios que obtiene a medias con el propietario, el cual paga las pocas inversiones qeu se hacen como, por ejemplo, la compra de abonos.

El aparcero utiliza aperos tradicionales y cultiva los productos típicos de la región: algarrobos, olivos, avellanos y viña. También cuida de un pequeño huerto para consumo familiar que riega con el agua de un pozo el cual a veces se seca en verano. Información sobre la finca en 2005

El nuevo propietario vive en el pueblo, es joven, con estudios. mucha iniciativa y le gusta la agricultura. Dispone de capital dado que su familia se dedica a la industria. Este joven agricultor explota directamente la tierra con la ayuda de dos jornaleros fijos y de algunos eventuales.

Además de comprar la finca, ha introducido cultivos muy inno. vadores, como una variedad de almendro que es un árbol de secano con pie de frutal de regadio. El propietario se arriesga con esta clase de aimendro ya que las técnicas que utiliza son muy nuevas; sabe que el rendimiento aumentará mucho y calcula que dentro de 8-10 años tendrá buenos beneficios. También ha adquirido acciones del pantano para disponer de agua, ha construido una balsa, ha instalado un sistema de riego automático y ha nivelado algunas parcelas para poder regar y utilizar maquinaria.

a) Lee los textos relacionados con el paso de una explotación agraria de economia tradicional a una economia de mercado y considera los planos de la finca. Después completa ampliamente los siguientes aspectos:

- Cambios en la propiedad.

- Cambios en la explotación de la tierra y en el trabajo.

- Cambios en la parcelación de las tierras.

- Cambios en los cultivos.

- Cambios en las técnicas de cultivo.

- Cambios en la capitalización.

- Cambios referentes a la comercialización.

Fuente: Albet y Benejam, 2009. 
Por último, el manual de Anaya, publicado en el año 2016 y actualizado a la ley educativa vigente, la LOMCE, organiza los contenidos en doce unidades didácticas. Además, incluye una introducción sobre la geografía, el espacio geográfico y fuentes geográficas, un apéndice cartográfico con mapas físicos y políticos de España y Europa, y un apéndice estadístico con datos de actividades económicas y población de España a nivel provincial. Del mismo modo que en caso de la editorial Vicens Vives, no contemplaron ninguna unidad didáctica específica para estudiar el medio rural. De los doce temas propuestos, uno de ellos está enfocado a estudiar los espacios del sector primario. Se trata de un tema voluminoso, en torno a cincuenta páginas y con muchos contenidos pero que, desafortunadamente $\mathrm{y}$, desde nuestro punto de vista, no esclarecen las nuevas realidades del medio rural actual.

Los contenidos de la unidad didáctica dedicada al sector primario gira en torno a los condicionantes naturales y humanos del espacio agrario, las actividades y paisajes agrarios, los problemas agrarios y el desarrollo rural, y los espacios de la actividad pesquera. Resulta curioso que en la introducción de esta unidad didáctica se haga referencia a que "El espacio rural es un territorio multifuncional que, junto a las actividades tradicionales, está implantando nuevas funciones industriales, terciarias y ambientales demandadas por una sociedad postmoderna" o que "Se convirtió en un espacio dinámico, complejo y heterogéneo [...] y donde la tradicional separación entre lo rural y lo urbano tiende a desvanecerse" (Muñoz, 2016, p. 146). Estas ideas parecen estar descontextualizadas en un tema que lleva por título "Los espacios del sector primario" y revelan la necesidad de crear una unidad didáctica propia en la que se expongan las transformaciones de estos espacios en las últimas décadas, así como las nuevas actividades que están acogiendo, en gran medida, fruto de la difusión de las tecnologías de la información y de las comunicaciones. Solo hay dos epígrafes, muy breves, donde se comentan algunas características del medio rural y sus dinámicas más recientes.

En cuanto a las actividades, este manual plantea una gran cantidad de ejercicios. A lo largo de los distintos epígrafes se incluyen cuarenta y siete tareas con el fin de ayudar a los discentes a reforzar lo aprendido pero que, por su naturaleza, implican una gran dedicación. En estas actividades se indica al alumnado que sintetice en cuadros los principales contenidos teóricos, y que busque información en diferentes fuentes para complementar dichos contenidos. Un ejemplo se puede observar en una de las actividades (Muñoz, 2016, p. 154) donde se solicita a los alumnos que consulten en un libro de historia cómo se llevaron a cabo las políticas de desamortización, la reforma agraria de la Segunda República, y la política de colonización del franquismo y valorar sus resultados. Aparte de estas, al final de la unidad didáctica, se proponen "Actividades de síntesis" que consisten en definir conceptos y realizar cuadros comparativos de síntesis - Figura 3. A nuestro juicio, esta gran cantidad de actividades, que requieren mucho esfuerzo por parte 
del alumnado, son desproporcionadas y, en su mayoría, están asociadas al modelo didáctico tradicional o transmisivo, donde se enseña una geografía descriptiva y memorística en lugar de promover una geografía activa, reflexiva y crítica (Macía, Rodríguez y Armas, 2015).

Figura 3: Cuadro síntesis. Comparar la situación actual y las transformaciones recientes de la estructura agraria española.

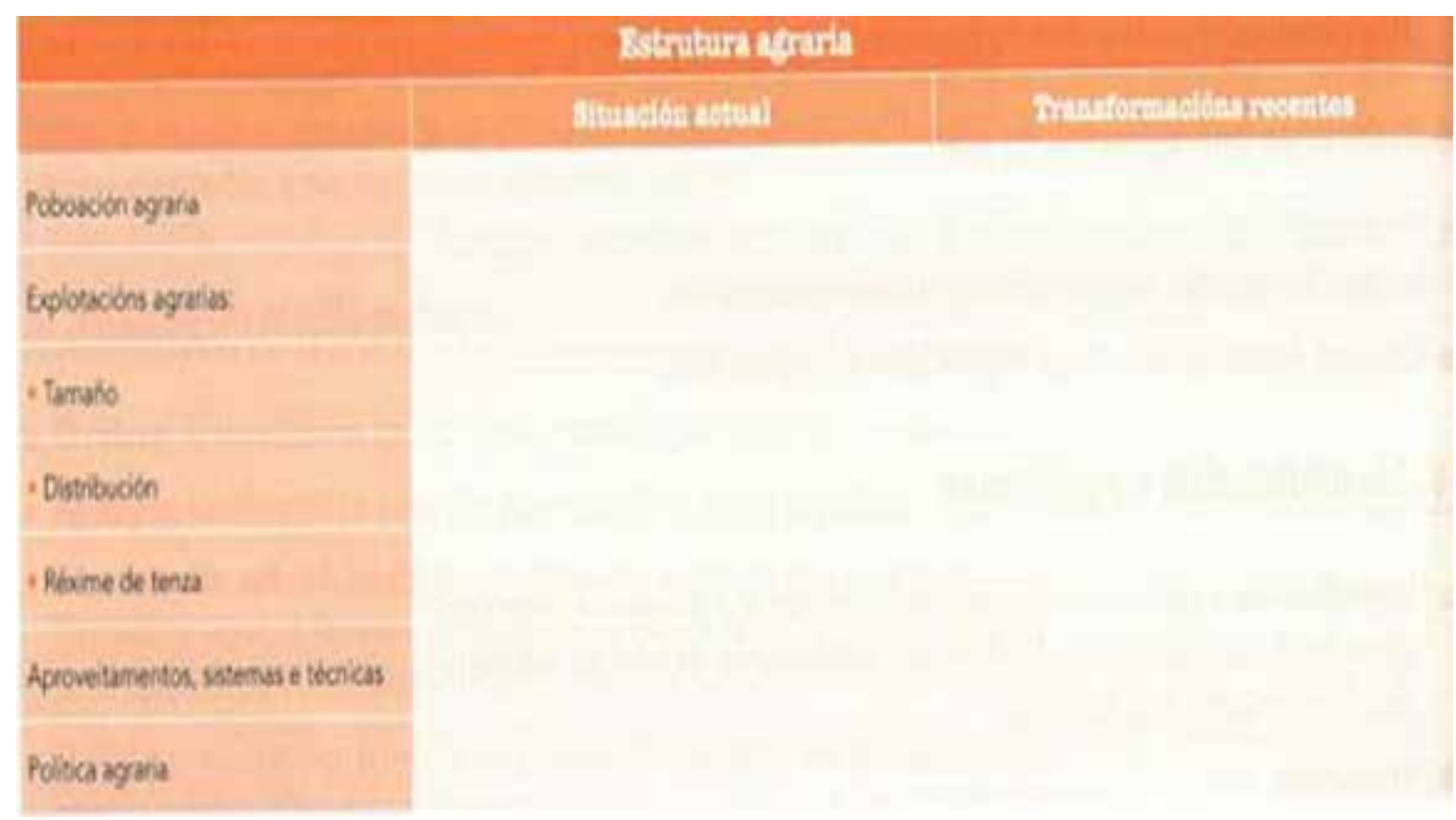

Fuente: Muñoz, 2016.

\section{Reflexiones finales}

El medio rural en Galicia, así como en España y Europa, ha experimentado grandes transformaciones tanto a nivel económico como social y cultural, que complican cada vez más la difícil tarea de definirlo y delimitarlo. La desaparición de las distancias en la sociedad de la información, así como la difusión de las nuevas tecnologías en estas áreas, está haciendo 
posible el establecimiento de nuevas actividades que, en el pasado, eran exclusivas de los ámbitos urbanos. Este proceso está dando lugar a diferentes grados de ruralidad, con distintos escenarios, dinámicas y problemas que deben ser expuestas, analizadas y aprehendidas por el alumnado. Ya no se puede asociar, en los países desarrollados y de forma exclusiva, lo rural a lo agrario, sino que el abanico de actividades crece cada día.

El resultado del análisis curricular para la materia de Geografía que se imparte en el segundo curso de bachillerato reveló que la visión predominante del medio rural, si no la única, es su fuerte vinculación con el sector primario. Del mismo modo, los libros de texto analizados adolecen, en general, de las mismas debilidades que el currículo y que, además de la escasez de contenidos, buena parte de las actividades que se plantean no fomentan un modelo de enseñanzaaprendizaje reflexivo y crítico. Tanto los contenidos curriculares como los que se reflejan en los libros de texto, omiten todos aquellos aspectos que no tienen relación con este sector primario y, curiosamente, son estos los que definen al medio rural del nuevo milenio.

\section{REFERENCIAS}

ALBET MAS, A.; BENEJAM ARGUIMBAU, P. Geografía. Barcelona: Vicens Vives, 2009.

ARMESTO LÓPEZ, X.A. Notas teóricas en torno al concepto de postproductivismo agrario. Investigaciones Geográficas, 36, pp. 137-156, 2005.

CAIRNCROSS, F. The death of distance 2.0. How the communications revolution will change our lives. London: Texere, 2001.

CALATRAVA, A.; MELERO, A. Aproximación a la estructura y los modelos de diversificación de la actividad empresarial en municipios rurales: análisis y tendencias en regiones españolas. Eure. Revista Latinoamericana de Estudios Urbano-regionales, 29 (88), 2003. pp. 97-130.

CEÑA DELGADO, F. Planteamientos económicos del desarrollo rural: una perspectiva 
histórica. In: RAMOS, E., CRUZ, J. (Coords.). Hacia un nuevo sistema rural. Madrid: Ministerio de Agricultura, Pesca y Alimentación, 1995, pp. 91-129.

DECRETO 86/2015, do 25 de xuño, polo que se establece o currículo da educación secundaria obrigatoria e do bacharelato na Comunidade Autónoma de Galicia. Diario Oficial de Galicia, núm. 120 (29 de junio de 2015).

ENTRENA-DURÁN, F. La ruralidad en España: de la mitificación conservadora al neorrularismo. Cuadernos de desarrollo rural, 9 (69), 2012, pp. 39-65.

ESPAÑA. MINISTERIO DE AGRICULTURA, PESCA Y ALIMENTACIÓN. Libro Blanco de la agricultura y el desarrollo rural. Madrid: Ministerio de Agricultura, Pesca y Alimentación, 2003.

FLORENCIO CALDERÓN, A. El desarrollo local y el desarrollo rural: el papel de los pequeños municipios. In: PÉREZ RAMÍREZ, B.; CARRILLO BENITO, E. (Coords.). Desarrollo local: manual de uso. Madrid: ESIC, 2000. pp. 527-554.

GARCÍA RAMÓN, M. D.; TULLAS I PUJOL, A. F.; VALDOVINOS PERDICES, N. Geografía rural. Madrid: Síntesis, 2010.

GUIBERTAU CABANILLAS, A. Fortalezas y debilidades del modelo de desarrollo rural por los agentes locales. In: MÁRQUEZ FERNÁNDEZ, (Coord). Nuevos horizontes en el desarrollo rural. Madrid: Akal, 2002, pp. 87-104.

HERVIEU, B. El espacio rural europeo entre la ruptura y el desarrollo. In: RAMOS, E., CRUZ, J. (Coords.). Hacia un nuevo sistema rural. Madrid: Ministerio de Agricultura, Pesca y Alimentación, 1995, pp. 27-48.

LÁZARO ARAUJO, L. El desarrollo rural en el contexto de la Unión Europea. In: RAMOS, E.; CRUZ, J. (Coords.). Hacia un nuevo sistema rural. Madrid: Ministerio de Agricultura, Pesca y Alimentación, 1995, pp. 237-255.

LEY ORGÁNICA 1/1990, de 3 de octubre, de Ordenación General del Sistema Educativo. Boletín Oficial del Estado, núm. 238 (4 de octubre de 1990).

LEY ORGÁNICA 2/2006, de 3 de mayo, de Educación. Boletín Oficial del Estado, núm. 106 (4 de mayo de 2006).

LEY ORGÁNICA 8/2013, de 9 de diciembre, para la mejora de la calidad educativa. Boletín Oficial del Estado, núm. 295 (10 de diciembre de 2016). 
MACÍA ARCE, X.C.; RODRÍGUEZ LESTEGÁS, F.; ARMAS QUINTÁ, F.X. Innovación TIC en la formación del profesorado para educación secundaria: Un ejemplo de buenas prácticas a propósito del diseño e implementación de una unidad didáctica sobre paisaje y desarrollo sostenible en Europa. En LÓPEZ FACAL, R. Ciencias Sociales, educación y futuro. Investigaciones en didáctica de las ciencias sociales. Santiago de Compostela: Red-14-Universidade de Santiago de Compostela, 2016, pp. 1180-1195.

MACÍA ARCE, X. C., RODRÍGUEZ LESTEGÁS, F.; ARMAS QUINTÁ, F. X. Manual de aprendizaxe e ensinanza de Xeografia e Ciencias Sociais para Educación Secundaria. Santiago de Compostela: Andavira, 2015.

MÁRQUEZ FERNÁNDEZ, D. (Coord.). Nuevos horizontes en el desarrollo rural. Madrid: Akal, 2002.

MCDONAGH, J. Rurality and Development in Ireland, the need for debate? Irish Geography, 31, (1), 1998, pp. 47-54.

MOLINERO HERNANDO, F.; ALARIO TRIGUEROS, M. La delimitación geográfica del desarrollo rural: una perspectiva histórica. Revista de Estudios Agro-Sociales, 169, pp. 53-87, 1994.

MOYANO, E. Procesos de cambio en la sociedad rural española: Pluralidad de intereses en una nueva estructura de oportunidades. Papers: Revista de Sociología, 61, 2000, pp. 191-220.

MUÑOZ-DELGADO, Ma . C. (Coord.). Xeografia. Madrid: Grupo Anaya, 2001

MUÑOZ-DELGADO, Mª C. Xeografía. Madrid: Grupo Anaya, 2016.

PANIAGUA MAZORRA, A.; HOGGART, K. Lo rural, ¿Hechos, discursos o representaciones? Una perspectiva geográfica de un debate clásico. Información Comercial Española. Revista de Economía, 803, 2002, pp. 61-71.

PLESSIS, V. et al. Definitions of rural. In: MINISTER OF INDUSTRY OF CANADA. Agriculture and Rural Working. Paper Series, Working Paper 61, Ottawa: Minister of Industry of Canada, 2001, pp. 1-43.

PRATT, A. Discourses of rurality: Loose Talk or Social Struggle. Journal of Rural Studies, 12 (1), 1996, pp. 69-78.

REAL DECRETO 1105/2014, de 26 de diciembre, por el que se establece el currículo básico de 
la Educación Secundaria Obligatoria y del Bachillerato. Boletín Oficial del Estado, núm. 3 (3 de enero de 2015).

ROBINSON, G.M. Conflict and change in the countryside. London: Belhaven Press, 1990.

RUBIO TERRADO, P. Modelización de los cambios y evolución reciente del sistema rural español. Boletín de la Asociación de Geógrafos Españoles, 54, pp. 203-235, 2010.

SANCHO COMÍNS, J.; REINOSO MORENO, D. La delimitación del ámbito rural: una cuestión clave en los programas de desarrollo rural. Estudios Geográficos, 73 (273), pp. 599-624, 2012.

SOUTO GONZÁLEZ, X. M. Didáctica de la Geografia. Problemas sociales y conocimiento del medio. Barcelona: Ediciones del Serbal, 1998. 